\title{
Painless pulsatile mass as first presentation of renal cell carcinoma
}

\author{
Kimberley Hoyland ${ }^{1}$, Nikhil Vasdev' ${ }^{1}$ Paul Nathan ${ }^{2}$, Damian Hanbury ${ }^{1}$ \\ ${ }^{I}$ Department of Urology, Hertfordshire and South Bedfordshire Urological Robotic Cancer Centre, Lister Hospital, UK. \\ ${ }^{2}$ Department of Oncology, Hertfordshire and South Bedfordshire Urological Robotic Cancer Centre, Lister Hospital, UK.
}

Received March 15, 2014; Revised May 08, 2014; Accepted May 10, 2014; Published Online May 22, 2014

\section{Case Report}

\begin{abstract}
Renal cell carcinoma rarely presents with typical features of abdominal pain, flank mass and haematuria, and up to $30 \%$ of patients present with metastases. We present the case of an 81 year old man who presented with a painless, pulsatile sternal metastasis as an initial presentation of renal cell carcinoma. This case highlights the importance of recognizing even unusual presentations of renal cell carcinoma, and that although sternal metastases are rare, when they do occur there should be a low threshold for ruling out renal cell carcinoma.
\end{abstract}

Keywords: Renal Cell Carcinoma; Sternal mass; Outcome

\section{Introduction}

Renal cell carcinoma is the eighth most common cancer in the UK, accounting for $3 \%$ of all new cases. ${ }^{1}$ However, the typical presentation of abdominal pain, flank mass and haematuria, only occurs in around $10 \%$ of patients. ${ }^{2}$ Furthermore, up to $39 \%$ of patients are completely asymptomatic at presentation, and $25-30 \%$ present with metastases. ${ }^{2}$ The prognosis for renal cell carcinoma with metastases is poor ${ }^{2}$, with only $10 \%$ survival at 1 year. ${ }^{3}$ We present here a case report of a patient presenting with a sternal metastasis as an initial presentation of renal cell carcinoma.

\section{Case Presentation}

An 81 year old gentleman presented via cardiology for a routine assessment for a Transcatheter Aortic Valve Implantation. During the assessment the patient was found to have a large, abnormal, soft tissue mass overlying the sternum. On further questioning the patient reported noticing the lump a few weeks previously, but did not recall any history of trauma to this area. The patient reported no pain or discomfort

Corresponding author: Nikhil Vasdev; Department of Urology, Hertfordshire and South Bedfordshire Urological Robotic Cancer Centre, Lister Hospital, UK.

Cite this article as:

Hoyland K, Vasdev N, Nathan P, Hanbury D. Painless pulsatile mass as first presentation of renal cell carcinoma. Int J Cancer Ther Oncol 2014; 2(3):02036. DOI: 10.14319/ijcto.0203.6 associated with the lump, and was unsure if it had altered in size or shape. Following additional questioning, the patient alluded to suffering from abdominal bloating, poor appetite and constipation for the preceding months. However, he had no symptoms of abdominal pain, weight loss, urinary symptoms or hematuria. The patient's background included aortic stenosis, hypertension, type 2 diabetes and a cerebrovascular event.

On examination the patient had a soft tissue mass overlying the sternum, approximately $12 \mathrm{~cm}$ in size. The border was irregular, and the mass was expansile and pulsatile. The mass was fixed to the underlying structures. The patient's chest was clear, except a moderate ejection systolic murmur. The only finding on examination of the abdomen was fullness in the right flank. However, there were no palpable abdominal masses or lymphadenopathy.

\section{Investigation}

A CT scan revealed a large destructive and expansile lesion replacing most of the body of the sternum. Peripherally, the sternal mass revealed increased vascularity, with low attenuation centrally. In addition to this the CT scan showed a large mass at the superior aspect of the right kidney, approximately $11.5 \mathrm{~cm}$ in the axial diameter. There was no lymph node involvement or bony lesions elsewhere. 

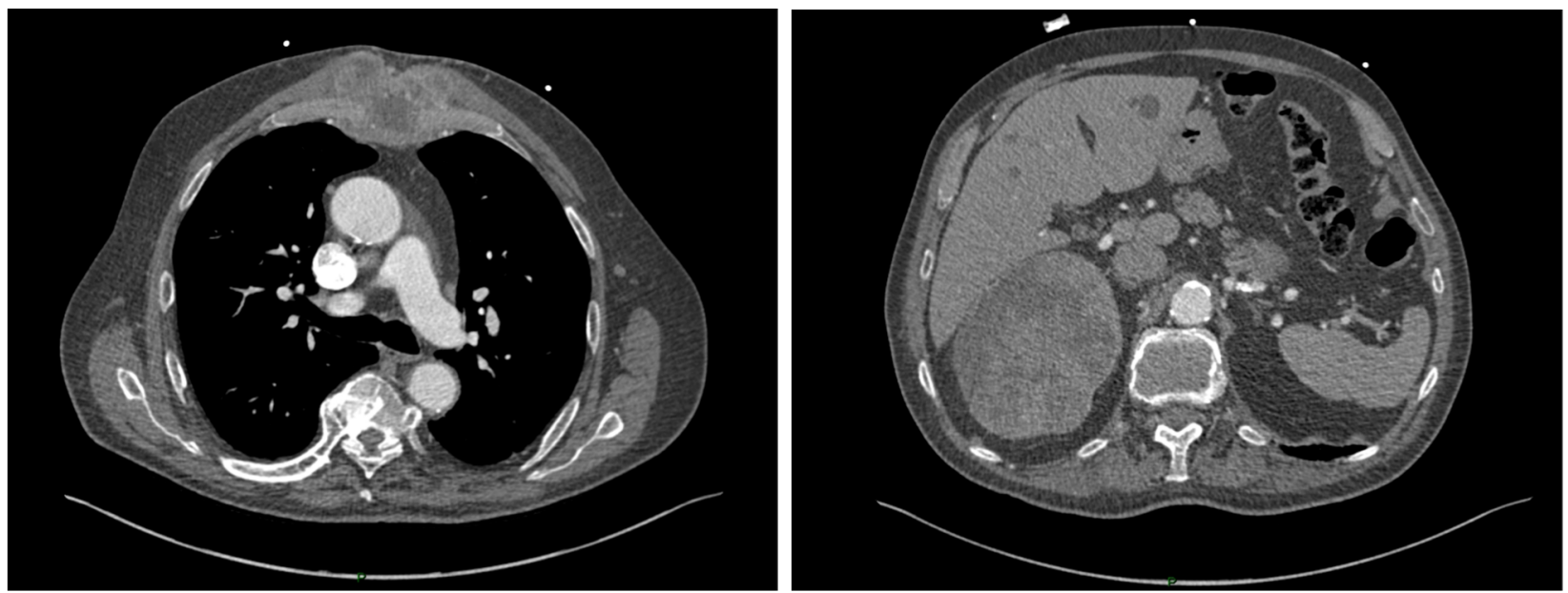

FIG. 1: (Left): Sternal metastasis seen on CT scan of thorax. (Right): Mass at superior aspect of right kidney measuring $11.5 \mathrm{~cm}$ in axial diameter.

\section{Differential Diagnosis}

Without histological diagnosis, the differential diagnoses for a chest wall mass are mainly neoplastic, infective or vascular. The mass was therefore likely to be either a tumour or metastasis, a hematoma or an abscess. Given that inflammatory markers were not raised, and the patient was afebrile, infection was ruled out. Hematoma was highly unlikely given that there was no history of trauma or anticoagulant therapy. Furthermore, the radiological findings were against both vascular and infective causes. Therefore given the findings of the right renal mass, the highest probable cause was neoplastic.

\section{Outcome and Follow Up}

Unfortunately, due to the patients' co-morbidities and fragile state, it was not possible to obtain a biopsy of the sternal lesion. Renal cell carcinoma metastases are renowned to be highly vascular, presenting a high risk of bleeding if biopsy were to be performed, and this would have compromised the patients' already poor health. In addition, the patient was not fit for surgical removal or treatment of the RCC or the metastasis, and thus it was not possible to obtain any histological specimen. This presents a diagnostic dilemma, due to the lack of histological evidence for a sternal metastasis, however, CT appearances were convincing of a metastasis, and there was no further history or evidence to suggest an alternative differential diagnosis.

\section{Discussion}

Sternal metastases are a rare presentation of renal cell carcinoma, with only a handful reported in the literature. ${ }^{1-8}$ However, sternal metastases have a high likelihood of being renal in origin, with over $75 \%$ being of either renal or thy- roid origin. ${ }^{4}$ In contrast, primary sternal tumours are very rare, and thus an alternative origin should be sought. ${ }^{4}$ Cases of sternal metastases have been reported since the early 1900 's, with many found to have primary carcinomas in the kidney; for example Eshner 1908, MacLeod and Jacobs 1921, Crile 1936, Roth and Davidson 1937, and Fredell and Stone 1956.5

The majority of cases reported, refer to sternal metastases, which present with retrosternal or anterior chest discomfort. ${ }^{1-8}$ However, this patient was unusual in that he presented incidentally, and had no complaints of pain or discomfort, despite a large mass in the sternum.

Treatment options are limited for metastatic disease, as less than $5 \%$ respond to chemotherapy, making the prognosis poor. $^{2}$ There are a few cases reported in the literature of patients with sternal metastases and renal cell carcinoma who underwent nephrectomy and sternal resection, which resulted in patients being asymptomatic up to 5 years postoperatively. ${ }^{6}$ We also had another patient who had a nephrectomy for renal cell carcinoma, as well as radiotherapy for a sternal metastasis, diagnosed histologically following biopsy, and the patient has been recurrence free for the last eight years. Although this has been shown to potentially improve survival and quality of life ${ }^{7}$, unfortunately our current patient was unfit for treatment due to pre-existing co-morbidities, and has now sadly passed away.

\section{Conclusion}

- $\quad$ RCC is fairly common and rarely presents with typical symptoms.

- Clinicians should be aware of the wide range of possible metastases from RCC, including sternal metastases. 
- Sternal metastases, although rare, have been reported in the literature, and if present one should attempt to rule out RCC as a cause.

- $\quad$ For solitary sternal metastases, literature suggests sternal resection and nephrectomy may increase survival.

\section{Conflict of interest}

The authors declare that they have no conflicts of interest. The authors alone are responsible for the content and writing of the paper.

\section{References}

1. http://www.cancerresearchuk.org/cancer-info/canc erstats/types/kidney/incidence/

[Accessed: December 19, 2012].

2. Batista RR, Marchiori E, Takayassu TC, et al. Sternal metastasis as an initial presentation of renal cell carcinoma: a case report. Cases J 2009; 2:9045.

3. Ngai H-Y, Ho L-Y, Chan T-F, et al. Rare presentation of renal cell carcinoma: Solitary sternal metastasis. Surgical Practice 2008; 12: 99-101.

4. Estrera AS, Platt MR, Mills LJ, Shaw RR. Pulsatile sternal tumor: report of three cases and a review of the literature. Ann Thorac Sug1981; 31: 244-50.

5. Fredell EW, Stone AO. Pulsating lesions metastatic from renal cancer. Calif Med 1956; 85: 93-6.

6. Pyle JW, Ash JL, Hussain SM, et al. Sternal resection and reconstruction after renal cell carcinoma metastatic to the sternum. I Thorac Cardiovasc Surg 2005; 129:1177-8.

7. Lee SY, Lee SJ, Lee CS. Sternum resection and reconstruction for metastatic renal cell cancer. Int $J$ Surg Case Rep 2011; 2:45-6.

8. Baltic V. Rare solitary sternal metastasis of renal cell carcinoma. Arch Oncol 2008; 16:13. 